\title{
Polymorphisms in the PE35 and PPE68 antigens in Mycobacterium tuberculosis strains may affect strain virulence and reflect ongoing immune evasion
}

\author{
YI JIANG $^{1 *}$, JIANHAO WEI $^{1,2^{*}}$, HAICAN LIU $^{1 *}$, GUILIAN LI $^{1 *}$, QIAN GUO ${ }^{1}$, YAN QIU ${ }^{1}$, \\ LILI ZHAO $^{1}$, MACHAO LI ${ }^{1}$, XIUQIN ZHAO ${ }^{1}$, XIANGFENG DOU ${ }^{3}$ and KANGLIN WAN ${ }^{1}$ \\ ${ }^{1}$ Department of Tuberculosis, State Key Laboratory for Infectious Disease Prevention and Control, \\ Collaborative Innovation Center for Diagnosis and Treatment of Infectious Diseases, \\ National Institute for Communicable Disease Control and Prevention, Chinese Center for Disease Control and Prevention, \\ Beijing 102206; ${ }^{2}$ Department of Laboratory Medicine, Shanghai Public Health Clinical Center, \\ Affiliated to Fudan University, Shanghai 201508; ${ }^{3}$ Institute for Infectious Disease Prevention and Control, \\ Beijing Center for Disease Prevention and Control, Beijing 100013, P.R. China
}

Received March 17, 2015; Accepted October 29, 2015

DOI: $10.3892 / \mathrm{mmr} .2015 .4589$

\begin{abstract}
Previous studies have demonstrated that the Pro-Glu/Pro-Pro-Glu (PE/PPE) genes in strains of Mycobacterium tuberculosis exhibit high sequence variation and may be involved in antigenic variation and immune evasion. Region of Difference 1 (RD1), encoding genes from Rv3871 to Rv3879, was observed to be lost during the original derivation of Bacillus Calmette-Guérin between 1908 and 1921. It has been previously demonstrated that two PE/PPE proteins, PE35 (Rv3872) and PPE68 (Rv3873), are encoded by RD1 and exhibit immunodominance. To explore the genetic diversity of PE35 and PPE68, and to evaluate the impact of sequence variation on the immune recognition of these proteins, 161 clinical M. tuberculosis strains were selected from China and comparative sequence analysis of PE35 and PPE68 was performed. The results indicated that polymorphisms in PE35 and PPE68 may lead to alterations in the function of these proteins, which may potentially affect strain virulence. In addition, the human T-cell epitopes of PE35 and PPE68 were highly variable, suggesting that the two antigens may be involved in diversifying selection to evade
\end{abstract}

Correspondence to: Dr Kanglin Wan, Department of Tuberculosis, State Key Laboratory for Infectious Disease Prevention and Control, Collaborative Innovation Center for Diagnosis and Treatment of Infectious Diseases, National Institute for Communicable Disease Control and Prevention, Chinese Center for Disease Control and Prevention, P.O. Box 5, Changping, Beijing 102206, P.R. China E-mail: wankanglin@icdc.cn

*Contributed equally

Key words: polymorphism, Mycobacterium tuberculosis, PE35, PPE68 host immunity. The prevalence of strains with PE35 mutations in the non-Beijing family was significantly greater compared with the Beijing family, indicating that Beijing strains may be more conservative than non-Beijing strains in this gene.

\section{Introduction}

Comparative genomics has identified numerous genetic regions in Mycobacterium tuberculosis and M. bovis that are deleted in $M$. bovis Bacillus Calmette-Guérin (BCG), such as region of difference 1 (RD1) and RD2 (1). RD1 was lost during the original derivation of BCG between 1908 and 1921 (2). Proteins encoded in these regions have the potential to form the basis of novel specific T-cell-based blood tests that do not cross-react with BCG. Among these antigens, early secretory antigenic target 6 (ESAT-6; ESXA, Rv3875), ESAT-6-like protein esxB (CFP10; ESXB, Rv3874), Pro-Pro-Glu 68 (PPE68; Rv3873) and Pro-Glu 35 (PE35; Rv3872) are immunodominant (3-5). The former two antigens (ESAT-6 and CFP10) have been investigated in detail in humans and are known to be predominant virulence factors $(6,7)$ and in addition are good candidates for the diagnosis of tuberculosis (TB) (8). The latter two, PE35 and PPE68, are members of the PE/PPE family and exhibit immunodominance (9). The PE/PPE proteins are secreted or associated with the mycobacterial cell envelope, and mediate interactions at the host-pathogen interface (10-12). PE35 and PPE68 have been demonstrated to be associated with cellular immune responses to mycobacterial infections (9).

Numerous previous studies have demonstrated high sequence variation of $\mathrm{PE} / \mathrm{PPE}$ genes in $M$. tuberculosis strains $(13,14)$, an observation that suggests involvement in antigenic variation. To improve the understanding of the genetic diversity of PE35 and PPE68 belonging to the PE-PPE genes in the RD1 region, and to explore the effect of immune recognition on the sequence variation of these two genes, the current study selected 161 clinical M. tuberculosis isolates in China, amplified the PE35 and PPE68 genes and compared 
the sequences. The effect of the polymorphisms in PE35 and PPE68 were investigated at the protein level to identify whether alterations in the function of these proteins occurs as this may potentially affect strain virulence. In addition, the variation in the human T-cell epitopes of PE35 and PPE68 were investigated to explore whether the two antigens are involved in diversifying selection to evade host immunity.

\section{Materials and methods}

Ethics statement. The study obtained approval from the Ethics Committee of National Institute for Communicable Disease Control and Prevention, Chinese Center for Disease Control and Prevention (Beijing, China). The patients with TB included in the present study were provided with a subject information sheet and written informed consent was obtained.

Strains and DNA preparation. A total of 161 strains were selected from 2,346 M. tuberculosis complex (MTBC) strains isolated in Beijing Municipality and 12 provinces and autonomous regions in China (Table I), which were genotyped by spoligotyping as described previously (15-18). Strains belonging to all major and rare spoligotypes in China were included. Considering the predominance of the Beijing family strains in China, approximately half of the Beijing family strains (82 strains) and half non-Beijing family strains (79 strains) were selected. The 82 Beijing family strains were randomly selected from the 1,738 Beijing strains among the total 2,346 strains. The remaining 79 strains were selected from 608 non-Beijing family isolates. Furthermore, it was attempted to include strains representing different spoligotypes that were isolated from different locations. Table I presents the numbers of strains used in the present study that were obtained from different provinces in China. The spoligotype patterns of the 161 strains are presented in Table II.

A total of 2,346 M.tuberculosis isolates were randomly collected between 2005 and 2007 from 2,346 patients at 13 different provincial tuberculosis hospitals across China (16). Subsequently, 161 strains were selected from those 2,346 isolates. Sputum specimens were collected from the TB patients and used to inoculate Löwenstein-Jensen slants. The strains were cultured using a standard Löwenstein-Jensen medium (Baso Diagnostics, Inc., Zhuhai, China) method (15), heat inactivated and then used directly in polymerase chain reactions (PCRs).

Primers. The nucleotide sequences of the primers used in the present study were designed with DNASTAR software (version 7.0; DNASTAR, Inc., Madison, WI, USA) according to the M. tuberculosis $\mathrm{H} 37 \mathrm{Rv}$ genomic sequence and were as follows: PE35, forward 5'-GTAATCGAGTTCGGGCAA TG-3' and reverse 5'-AGGCTTCTCCCAGAGAGTT-3'; PPE68, forward 5'-GACATTGGCACGCAAGTGAG-3' and reverse 5'-TAGCGGCATCGGTCTTCATC-3'.

PCR. The PCRs were performed in a total volume of $20 \mu \mathrm{l}$. The PCR mix contained $10 \mu 1$ PCR buffer (Tiangen Biotech (Beijing) Co., Ltd., Beijing, China), $100 \mathrm{nM}$ primer, $200 \mu \mathrm{M}$ each of the four dNTPs (Tiangen (Beijing) Co., Ltd.) and
Table I. Number of strains in different locations in China.

\begin{tabular}{lc}
\hline Location & Number of isolates \\
\hline Anhui & 11 \\
Shanxi & 16 \\
Beijing & 11 \\
Fujian & 24 \\
Gansu & 12 \\
Guangxi Zhuang Autonomous Region & 29 \\
Sichuan & 1 \\
Henan & 12 \\
Hunan & 7 \\
Xizang (Tibet) Autonomous Region, & 4 \\
Xinjiang Uygur Autonomous Region & 11 \\
Jilin & 12 \\
Zhejiang & 11 \\
\hline
\end{tabular}

Table II. Number of strains of each spoligotype pattern.

\begin{tabular}{lc}
\hline Spoligotype & Number of strains \\
\hline Beijing & 82 \\
T & 12 \\
U & 27 \\
MANU & 10 \\
Haarlem & 4 \\
EAI & 2 \\
LAM & 2 \\
S & 1 \\
CAS & 3 \\
New & 18
\end{tabular}

MANU, a new family from India; LAM,Latin-American-Mediterranean lineage; EAI, East African-Indian lineage; CAS, Central-Asian lineage; S, Sicily and Sardinia family; U, Ural region; T, modern tuberculosis strains.

0.5 units DNA Taq Polymerase (Takara Bio, Inc., Otsu, Japan). An initial denaturation of $5 \mathrm{~min}$ at $94^{\circ} \mathrm{C}$ was followed by 35 cycles of denaturation at $94^{\circ} \mathrm{C}$ for $45 \mathrm{sec}$, annealing at $62^{\circ} \mathrm{C}$ for $45 \mathrm{sec}$ and extension at $72^{\circ} \mathrm{C}$ for $1 \mathrm{~min}$, followed by a final extension at $72^{\circ} \mathrm{C}$ for $10 \mathrm{~min}$ in a Bio-Rad Thermal Cycler (Bio-Rad Laboratories, Inc., Hercules, CA).

Negative controls (reagents only, no DNA) were included each time the PCR was performed. The positive control was 500 pg DNA from the M. tuberculosis reference strain H37Rv. The presence and size of each PCR product was determined by electrophoresis on $2 \%$ agarose gel in Tris/boric acid/ethylenediaminetetraacetic acid buffer (Tiangen Biotech (Beijing) Co., Ltd.) followed by staining with ethidium bromide (SBS Genetech Co., Ltd., Beijing, China).

The PCRs were conducted a minimum of two times to validate the reproducibility. The variants were confirmed by the sequencing of the new PCR products. 
Table III. Comparison of the Mycobacterium tuberculosis strains containing mutations in the Beijing family and the non-Beijing family.

Strain Beijing family Non-Beijing family

Strains with PE35 mutation

Strains with PPE68 mutation

All

$\begin{array}{cc}4 & 19^{\mathrm{a}} \\ 2 & 6^{\mathrm{b}} \\ 82 & 79\end{array}$

${ }^{\mathrm{a}} \mathrm{P}=0.001$; Pearson $\chi^{2}$ test, Beijing strains vs. non-Beijing strains. ${ }^{\mathrm{b}} \mathrm{P}=0.253$; Pearson $\chi^{2}$ test, Beijing strains vs. non-Beijing strains. PE35, Pro-Glu 35; PPE68, Pro-Pro-Glu 68.
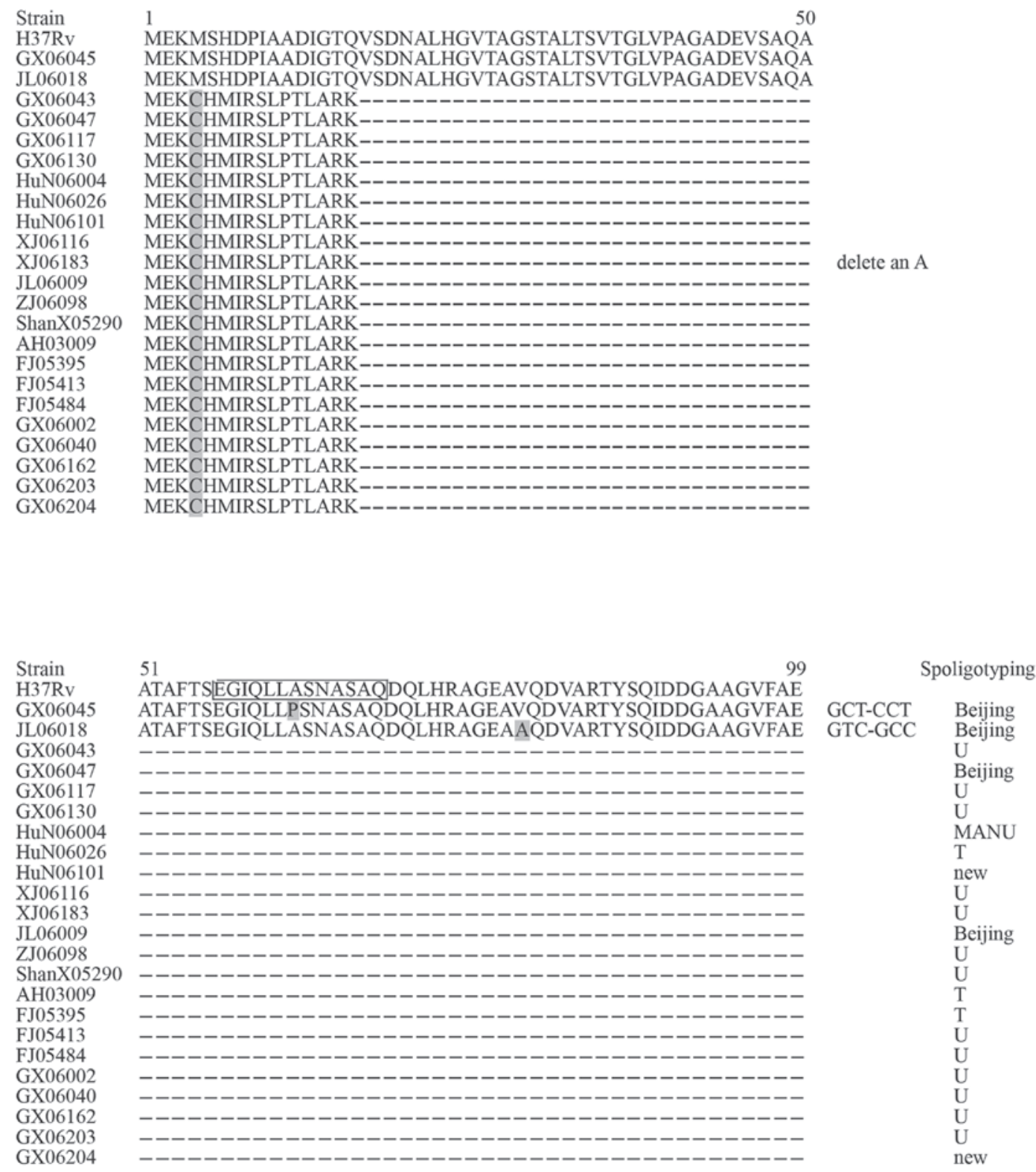

Figure 1. Sequence alignment of the PE35 antigen of the 8 strains of Mycobacterium tuberculosis exhibiting alterations. Of the total 161 strains, 23 strains exhibited alterations at the gene level whilst the remaining 138 stains were identical to the H37Rv strain. The T-cell epitope areas are boxed in H37Rv. Shading indicates the location the of amino acid alterations. PE35, Pro-Glu 35. MANU, a new family from India; U, Ural region.

Sequence and data analysis. The sequences of the PCR products were determined using an ABI 3730xl DNA Analyzer (Applied Biosystems; Thermo Fisher Scientific, Inc., Waltham, MA, USA).
The sequences were first aligned using ClustalW software (19) with the PE35 and PPE68 gene sequences from the M. tuberculosis H37Rv genome to determine the PE35 and PPE68 region. Sequence comparisons and translations were 


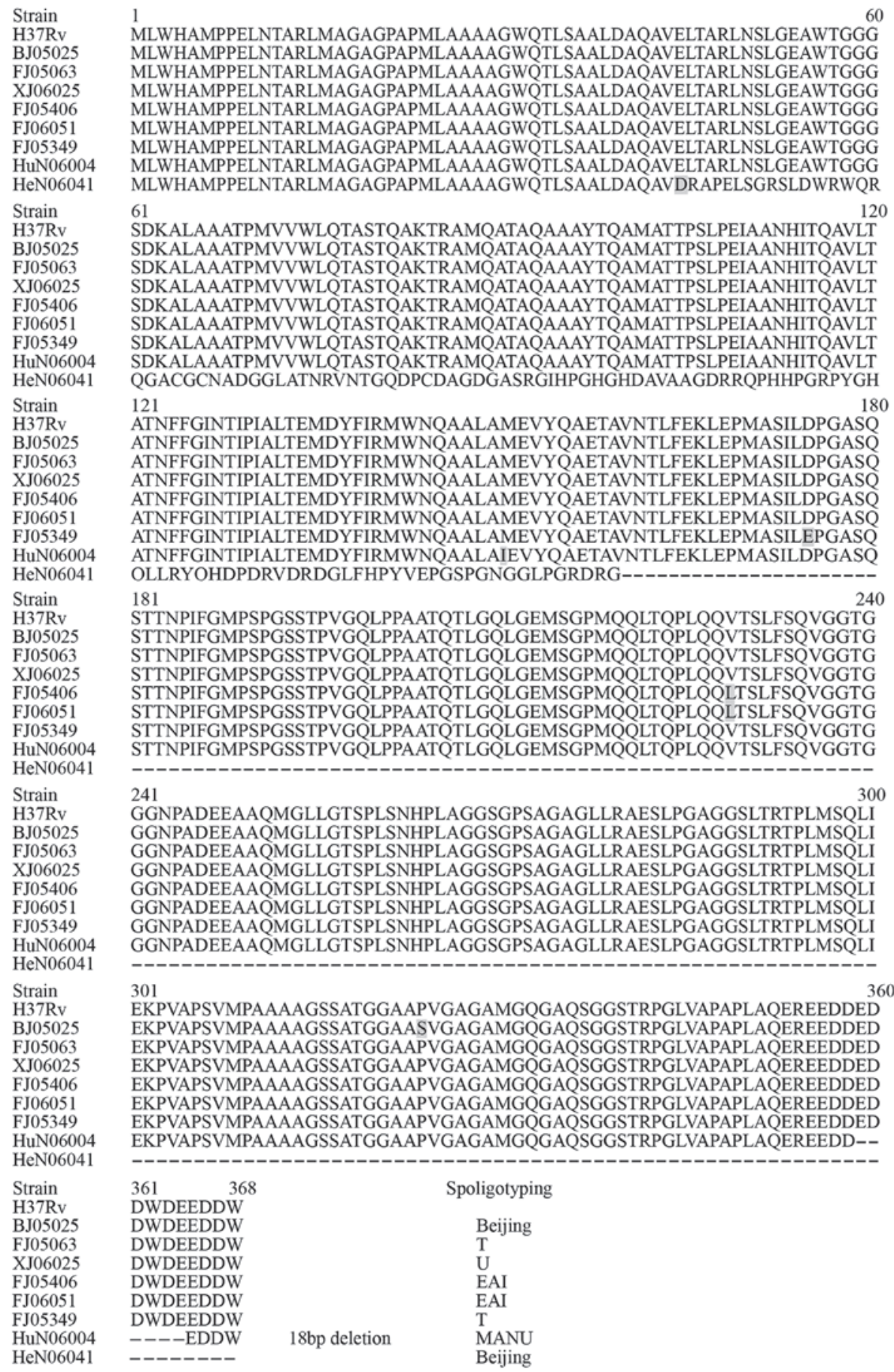

Sbp deletion

GAT-AAT

GAT-AAT

GTG-CTG

GTG-CTG

CCG-TCG

Figure 2. Sequence alignment of the PPE68 antigen in the 8 strains of Mycobacterium tuberculosis exhibiting alterations. Of the total 161 strains, 8 strains exhibited alterations at the gene level and the remaining 153 stains were identical to the H37Rv strain. Shading indicates locations of amino acid alterations. PPE68, Pro-Pro-Glu 68.

conducted using Bioedit software, version 7.1.3.0 (20). The Immune Epitopes Database (IEBD) (http://www.iedb.org/) was used and 1 human T-cell epitope in PE35 and 62 in PPE68 were found (21). In addition, SPSS software, version 14.0 (SPSS, Inc., Chicago, IL, USA) was used to conduct $\chi^{2}$ analysis. $\mathrm{P}<0.05$ was considered to indicate a statistically significant difference.

\section{Results}

Mutation and deletion in gene sequences. The genes encoding PE35 and PPE68 were amplified and the sequences compared.
All 161 strains yielded PCR products of these two antigens. Among the $161 \mathrm{M}$. tuberculosis strains, 23 isolates exhibited polymorphisms in the gene sequence of PE35 (Fig. 1) and 8 strains exhibited polymorphisms in PPE68. For PE35, there were 21 strains containing an A deletion and the remaining 2 strains harbored two different nonsynonymous mutations. For PPE68, two isolates had two different deletions and six strains showed five nonsynonymous mutations (Fig. 2).

Changes at the protein level. Figs. 1 and 2 present the amino acid (AA) alterations and their positions in the PE35 and 
Table IV. Amino acid alterations of human T-cell epitopes in the antigens, PE35 and PPE68 .

\begin{tabular}{|c|c|c|c|c|}
\hline Epitope ID & Epitope peptide sequence & Rv locus & Gene & Amino acid alteration \\
\hline 144881 & EGIQLLASNASAQ & Rv3872 & PE35 & GCT(A)-CCT(P); Frameshift \\
\hline 183 & AAGSSATGGAAPVGAGAMGQGAQSG & Rv3873 & PPE68 & CCG(P)-TCG(S); Frameshift \\
\hline 191 & AAGWQTLSAALDAQAVELTARLNSL & Rv3873 & PPE68 & Frameshift(5 bp deletion) \\
\hline 265 & AALAMEVYQAETAVNTLF & Rv3873 & PPE68 & ATG(M)-ATA(I); Frameshift \\
\hline 2434 & ALAMEVYQAETAVNTLFEKLEPMAS & Rv3873 & PPE68 & ATG(M)-ATA(I); Frameshift \\
\hline 2922 & ALTEMDYFIRMWNQAALAMEVYQAE & Rv3873 & PPE68 & ATG(M)-ATA(I); Frameshift \\
\hline 3098 & AMGQGAQSGGSTRPGLVA & Rv3873 & PPE68 & Frameshift \\
\hline 4186 & ARLMAGAGPAPMLAAAAG & Rv3873 & PPE68 & No change \\
\hline 4187 & ARLMAGAGPAPMLAAAAGWQTLSAA & Rv3873 & PPE68 & No change \\
\hline 4727 & ASQSTTNPIFGMPSPGSSTPVGQLP & Rv3873 & PPE68 & Frameshift \\
\hline 4969 & ATGGAAPVGAGAMGQGAQ & Rv3873 & PPE68 & CCG(P)-TCG(S); Frameshift \\
\hline 5063 & ATNFFGINTIPIAL & Rv3873 & PPE68 & Frameshift \\
\hline 11486 & EEAAQMGLLGTSPLSNHP & Rv3873 & PPE68 & Frameshift \\
\hline 14339 & ETAVNTLFEKLEPMASIL & Rv3873 & PPE68 & Frameshift \\
\hline 15812 & FFGINTIPIA & Rv3873 & PPE68 & Frameshift \\
\hline 16010 & FGMPSPGSSTPVGQLPPA & Rv3873 & PPE68 & Frameshift \\
\hline 18685 & GAMGQGAQSGGSTRPGLVAPAPLAQ & Rv3873 & PPE68 & Frameshift \\
\hline 18776 & GASQSTTNPIFGMPSPGS & Rv3873 & PPE68 & Frameshift \\
\hline 19860 & GGGSDKALAAATPMVVWLQTASTQA & Rv3873 & PPE68 & Frameshift \\
\hline 20016 & GGSGPSAGAGLLRAESLP & Rv3873 & PPE68 & Frameshift \\
\hline 20048 & GGTGGGNPADEEAAQMGL & Rv3873 & PPE68 & Frameshift \\
\hline 20997 & GLLGTSPLSNHPLAGGSGPSAGAGL & Rv3873 & PPE68 & Frameshift \\
\hline 21179 & GLVAPAPLAQEREEDDEDDWDEEDD & Rv3873 & PPE68 & 18 bp deletion; Frameshift \\
\hline 21707 & GPMQQLTQPLQQVTSLFS & Rv3873 & PPE68 & GTG(V)-CTG(L); Frameshift \\
\hline 22351 & GSGPSAGAGLLRAESLPGAGGSLTR & Rv3873 & PPE68 & Frameshift \\
\hline 22531 & GSSTPVGQLPPAATQTLGQLGEMSG & Rv3873 & PPE68 & Frameshift \\
\hline 22657 & GTGGGNPADEEAAQMGLLGTSPLSN & Rv3873 & PPE68 & Frameshift \\
\hline 29846 & KALAAATPMVVWLQTAST & Rv3873 & PPE68 & Frameshift \\
\hline 35251 & LDPGASQSTTNPIFG & Rv3873 & PPE68 & GAT(D)-AAT(N); Frameshift \\
\hline 35652 & LEPMASILDPGASQSTTN & Rv3873 & PPE68 & GAT(D)-AAT(N); Frameshift \\
\hline 35819 & LFEKLEPMASILDPGASQSTTNPIF & Rv3873 & PPE68 & GAT(D)-AAT(N); Frameshift \\
\hline 37727 & LLRAESLPGAGGSLTRTP & Rv3873 & PPE68 & Frameshift \\
\hline 38448 & LPEIAANHITQAVLTATN & Rv3873 & PPE68 & Frameshift \\
\hline 38492 & LPGAGGSLTRTPLMSQLIEKPVAPS & Rv3873 & PPE68 & Frameshift \\
\hline 39817 & LTATNFFGINTIPIA & Rv3873 & PPE68 & Frameshift \\
\hline 41291 & MDYFIRMWNQAALAMEVY & Rv3873 & PPE68 & ATG(M)-ATA(I); Frameshift \\
\hline 42095 & MLWHAMPPELNTARLMAG & Rv3873 & PPE68 & No change \\
\hline 46130 & NTIPIALTEMDYFIRMWN & Rv3873 & PPE68 & Frameshift \\
\hline 48567 & PMLAAAAGWQTLSAALDA & Rv3873 & PPE68 & No change \\
\hline 49875 & PVGQLPPAATQTLGQLGE & Rv3873 & PPE68 & Frameshift \\
\hline 50366 & QATAQAAAYTQAMATTPSLPEIAAN & Rv3873 & PPE68 & Frameshift \\
\hline 51367 & QLIEKPVAPSVMPAAAAGSSATGGA & Rv3873 & PPE68 & Frameshift \\
\hline 52167 & QQVTSLFSQVGGTGGGNP & Rv3873 & PPE68 & GTG(V)-CTG(L); Frameshift \\
\hline 52556 & QTLGQLGEMSGPMQQLTQ & Rv3873 & PPE68 & Frameshift \\
\hline 60492 & SQLIEKPVAPSVMPAAAA & Rv3873 & PPE68 & Frameshift \\
\hline 62250 & SVMPAAAAGSSATGGAAP & Rv3873 & PPE68 & CCG(P)-TCG(S); Frameshift \\
\hline 64822 & TLGQLGEMSGPMQQLTQPLQQVTSL & Rv3873 & PPE68 & Frameshift \\
\hline 65054 & TLSAALDAQAVELTARLN & Rv3873 & PPE68 & Frameshift (5 bp deletion) \\
\hline 65767 & TPSLPEIAANHITQAVLTATNFFGI & Rv3873 & PPE68 & Frameshift \\
\hline 65912 & TQPLQQVTSLFSQVGGTGGGNPADE & Rv3873 & PPE68 & GTG(V)-CTG(L); Frameshift \\
\hline 66074 & TRPGLVAPAPLAQEREED & Rv3873 & PPE68 & Frameshift \\
\hline 66364 & TSPLSNHPLAGGSGPSAG & Rv3873 & PPE68 & Frameshift \\
\hline
\end{tabular}


Table IV. Continued.

\begin{tabular}{llccc}
\hline Epitope ID & \multicolumn{1}{c}{ Epitope peptide sequence } & Rv locus & Gene & Amino acid alteration \\
\hline 68284 & VELTARLNSLGEAWTGGG & Rv3873 & PPE68 & Frameshift(5 bp deletion) \\
68285 & VELTARLNSLGEAWTGGGSDKALAA & Rv3873 & PPE68 & Frameshift (5 bp deletion) \\
69128 & VITMLWHAMPPELNTARLMAGAGPA & Rv3873 & PPE68 & Frameshift \\
69795 & VLTATNFFGINTIPIALT & Rv3873 & PPE68 & Frameshift \\
69796 & VLTATNFFGINTIPIALTEMDYFIR & Rv3873 & PPE68 & Frameshift \\
71944 & VWLQTASTQAKTRAMQAT & Rv3873 & PPE68 & Frameshift \\
71945 & VWLQTASTQAKTRAMQATAQAAAYT & Rv3873 & PPE68 & Frameshift \\
73833 & YFIRMWNQAALAMEV & Rv3873 & PPE68 & ATG(M)-ATA(I); Frameshift \\
121011 & TPMVVWLQTASTQAKTR & Rv3873 & PPE68 & Frameshift \\
144907 & IALTEMDYFIRMWNQAALAMEVY & Rv3873 & PPE68 & ATG(M)-ATA(I); Frameshift \\
144964 & TNFFGINTIPIALT & Rv3873 & PPE68 & Frameshift
\end{tabular}

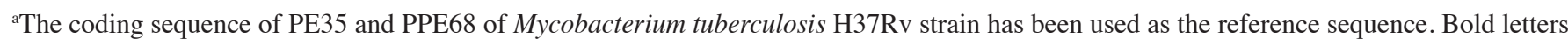
indicate locations of amino acid alterations. PE35, Pro-Glu 35; PPE68, Pro-Pro-Glu 68; ID, identification.

PPE68 antigens. All the alterations resulted in an AA change. A total of 21 strains with an A deletion in PE35 resulted in a frameshift, and therefore the premature termination of the protein, preventing it's production and thereby impacting upon protein function. HeN06041 contained a 5 base pair (bp) deletion located at the fifth AA of PPE68, which additionally resulted in premature termination, and may impact upon protein function due to the deletion abolishing the production of the protein. HuN06004 contained an 18 bp deletion, which resulted in a six AA deletion in PPE68.

Spoligotyping of variant strains. For PE35, 23 variant strains were identified, including 4 Beijing strains, $13 \mathrm{U}$ family strains, 3 T strains, 1 MANU strain and 2 new spoligotype strains. The two strains with nonsynonymous mutations were members of the Beijing family. For PPE68, 8 variant strains including 2 Beijing strains, $2 \mathrm{~T}$ strains, 2 EAI strains, $1 \mathrm{U}$ strain and 1 MANU strain were identified. The two EAI strains, FJ06051 and FJ05406, exhibited the same mutation in 229(V-L) in the AA sequence of PPE68, which may represent a unique mutation in EAI strains. HuN06004 exhibited polymorphisms in PE35 and PPE68.

The prevalence of strains containing a PE35 mutation in the non-Beijing family is significantly greater compared with the Beijing family (Table III, $\mathrm{P}<0.01$ ). The prevalence of strains with the PPE68 mutation in the non-Beijing family is greater compared with the Beijing family, however this was identified to be a significant difference.

Alterations in T-cell epitopes. There is 1 human T-cell epitope in PE35 and 62 in PPE68 according to the IEBD (http://www.iedb.org/) (21). Table IV presents the alterations in the T-cell epitopes of the two antigens. All mutations observed in PE35, except for that in JL06018, affected the T-cell epitopes. For PPE68, there were no nonepitope regions in the gene, as the 62 T-cell epitopes covered the whole gene sequence. This additionally indicates the importance of the PPE68 antigen for the development of T-cell immune responses following infection. Among all of the strains, 58/62 T-cell epitopes in PPE68
(93.5\%), exhibited AA alterations resulting from nucleotide alterations (Table IV).

The 5 bp deletion in HeN06041 resulted in a frameshift in the PPE68 protein code, leading to alterations in the corresponding T-cell epitopes including IEDB_ID 191, $65054,68284,68285$ and further downstream epitopes. The 18 bp deletion in HuN06004 resulted in a 6 AA deletion in IEDB_ID21179.

\section{Discussion}

In the present study, 161 clinical M. tuberculosis strains in China were selected which originated from a large geographical area and exhibited different spoligotyping patterns. This strategy was selected so that the data provided would be representative of the genetic diversity that may be present within China, at least to some extent.

In previous studies, genetic approaches coupled with biochemical analyses have indicated that proteins encoded by the RD1 locus are part of a secretion system required for ESAT-6 and CFP-10 export (22-26), hereafter referred to as the ESAT-6 system-1 (ESX-1). PE35 (Rv3872) and PPE68 (Rv3873) are encoded by RD1 and exhibited immunodominance (9). PE35 is an important antigen that stimulates human peripheral blood mononuclear cells in protective Th1 cell assays, demonstrating antigen-induced proliferation and $\gamma$-interferon secretion (4). PPE68 is predominantly associated with the cell wall (27) and forms complexes with the RD1 locus proteins Rv3866, Rv3868, CFP-10 and ESAT-6 $(28,29)$. A recent study (9) indicated that PE35 and PPE68 may serve a major role in RD1-associated pathogenesis, and may contribute to the establishment and maintenance of $M$. tuberculosis infection. Among the 161 strains investigated in the present study, $14.3 \%$ of strains with an A deletion in PE35 resulted in premature termination leading to a 16 AA peptide as opposed to the full length protein of 99 AA. This deletion would result in the prevention of protein production, and consequently lead to the complete loss of PE35 function. In addition, the 5 bp deletion in HeN06041 of PPE68 resulted in premature 
termination, and therefore may exert an effect on protein function via the abolition of protein production. Strains carrying mutations that lead to alterations in the functions of PE35 and PPE68 may be significantly compromised with regards to their virulence. Therefore, polymorphisms in PE35 and PPE68 may result in alterations in the functions of these proteins, which may potentially affect strain virulence. Furthermore, as PE35 has been demonstrated to be essential for ESXA/B secretion and RD1-mediated virulence (30), the null mutant of Rv3872 may influence the release of the ESX-1 antigens, CFP-10 and ESAT-6. PPE68 is a gating protein that regulates the release of ESX-1 antigens (30) therefore, abolition of PPE68 protein may additionally affect the release of CFP-10 and ESTA-6. To investigate this further, virulence comparison of mutant strains and wild strains of PE35 and PPE68 should be conducted.

PE/PPE genes are known to vary and to encode cell surface-exposed proteins, which has led to the hypothesis that they may be involved in antigenic variation (31) and have been suggested to be a 'molecular mantra' to aid in the escape of host immunity. Comas et al (32) reported that the human T-cell epitopes of $M$. tuberculosis are evolutionarily hyperconserved and suggested that M.tuberculosis lacks antigenic variation and immune evasion ability, however, the study excluded $\mathrm{PE} / \mathrm{PPE}$ genes. In the current study, there were 63 human T-cell epitopes identified in PE35 and PPE68 according to the IEDB (21). Among the strains, 59/63 T-cell epitopes (93.7\%), exhibited AA alterations resulting from nucleotide alterations. The large number of amino acid alterations in these T-cell epitopes may reflect ongoing immune evasion. The data from the current study supports the view that certain PE/PPE genes exhibiting high sequence variation may be involved in antigenic variation induced immune evasion.

The prevalence of strains with PE35 mutations in the non-Beijing family was significantly greater compared with the Beijing family, indicating that the Beijing family strains are less changeable in the T-cell epitopes of PE35 than the non-Beijing family strains. This is supported by a previous study, which demonstrated that Beijing strains from different geographic areas exhibited a high degree of genetic conservation compared with the other M. tuberculosis strains (33). There is evidence that T-cell responses may contribute directly to human-to-human transmission of MTBC (34). The current study indicated that the Beijing strains were more likely to be recognized by host T-cells in PE35 than the non-Beijing strains, which may render them easier to transmit than the non-Beijing strains. Furthermore, analysis of sequences of PE35 and PPE68 in isolates of these various lineages that have been isolated from non-Chinese populations could provide interesting information and insight.

In conclusion, it has been previously reported that PE35 had potential as a serodiagnostic candidate for M. tuberculosis (35). The results of the current study indicate that PE35 harbors a comparatively high number of AA alterations, suggesting that strain diversity should be considered during the further development of novel serodiagnostic candidates that contain PE35.

\section{Acknowledgements}

The current study was supported by the projects of the Natural Science Foundation of China (grant no. 81401647) and the
Chinese National Key Program of Mega Infectious Disease (grant nos. 2013ZX10003006 and 2013ZX10003002-001).

The authors would like to thank the staff at the institutes in the Beijing municipality, and the 13 provinces and autonomous regions in China for their contribution to the study, and in particular the help of Professor Lishui Zhang (Fuzhou Pulmonary Hospital of Fujian; Fujian, China), Professor Yunhong Tan (Hunan Chest Hospital; Hunan, China), Mr. Xiujun Yang (Jilin Institute for Tuberculosis Control; Jilin, China), Mrs. Chongxiang Tong (Gansu Institute for Tuberculosis Control; Gansu, China), Mrs. Feiying Liu (Guangxi Center for Disease Control and Prevention; Guangxi, China), Mr. Yingcheng Qi (Xinjiang Center for Disease Control and Prevention; Xinjiang, China), Professor Qing Wang (Anhui Chest Hospital; Anhui, China), Professor Xiaohui Cao (Haidian District Center for Disease Control and Prevention; Beijing, China), Professor Ping Zhao (Chaoyang District Center for Disease Control and Prevention; Beijing, China), Mr. Haitao Li (Henan Provincial Chest Hospital; Henan, China), Mrs. Jun Yang (Sichuan Center for Disease Control and Prevention; Sichuan, China), Mr. Xuanmin Zhang (Xi'an Chest Hospital; Xi'an, China), Professor Li Shi (Xizang Center for Disease Control and Prevention; Xizang, China) and Professor Xiaomeng Wang (Zhejiang Center for Disease Control and Prevention; Zhejiang, China).

\section{References}

1. Liu XQ, Dosanjh D, Varia H, Ewer K, Cockle P, Pasvol G and Lalvani A: Evaluation of T-cell responses to novel RD1- and RD2-encoded Mycobacterium tuberculosis gene products for specific detection of human tuberculosis infection. Infect Immun 72: 2574-2581, 2004.

2. Mahairas GG, Sabo PJ, Hickey MJ, Singh DC and Stover CK: Molecular analysis of genetic differences between Mycobacterium bovis BCG and virulent M. bovis. J Bacteriol 178: 1274-1282, 1996

3. Hanif SN, El-Shammy AM, Al-Attiyah R and Mustafa AS: Whole blood assays to identify Th1 cell antigens and peptides encoded by Mycobacterium tuberculosis-specific RD1 genes. Med Princ Pract 17: 244-249, 2008.

4. Mustafa AS, Al-Attiyah R, Hanif SN and Shaban FA: Efficient testing of large pools of Mycobacterium tuberculosis RD1 peptides and identification of major antigens and immunodominant peptides recognized by human Th1 cells. Clin Vaccine Immunol 15: 916-924, 2008.

5. Hanif SN, Al-Attiyah R and Mustafa AS: Species-specific antigenic Mycobacterium tuberculosis proteins tested by delayed-type hypersensitivity response. Int J Tuberc Lung Dis 14: 489-494, 2010.

6. Pym AS, Brodin P, Brosch R, Huerre M and Cole ST: Loss of RD1 contributed to the attenuation of the live tuberculosis vaccines Mycobacterium bovis BCG and Mycobacterium microti. Mol Microbiol 46: 709-717, 2002.

7. Lewis KN, Liao R, Guinn KM, Hickey MJ, Smith S, Behr MA and Sherman DR: Deletion of RD1 from Mycobacterium tuberculosis mimics bacille Calmette-Guérin attenuation. J Infect Dis 187: 117-123, 2003.

8. Malaghini M, Thomaz-Soccol V, Probst CM, Krieger MA, Preti H, Kritski A and Soccol CR: Recombinant antigen production for assays of intradermoreaction for diagnosis and surveillance of tuberculosis. J Biotechnol 156: 56-58, 2011.

9. Tiwari B, Soory A and Raghunand TR: An immunomodulatory role for the Mycobacterium tuberculosis region of difference 1 locus proteins PE35 (Rv3872) and PPE68 (Rv3873). FEBS J 281: 1556-1570, 2014.

10. Mukhopadhyay S and Balaji KN: The PE and PPE proteins of Mycobacterium tuberculosis. Tuberculosis (Edinb) 91: 441-447, 2011.

11. Sampson SL: Mycobacterial PE/PPE proteins at the host-pathogen interface. Clin Dev Immunol 2011: 497203, 2011. 
12. Akhter Y, Ehebauer MT, Mukhopadhyay S and Hasnain SE: The $\mathrm{PE} / \mathrm{PPE}$ multigene family codes for virulence factors and is a possible source of mycobacterial antigenic variation: Perhaps more? Biochimie 94: 110-116, 2012.

13. McEvoy CR, Cloete R, Müller B, Schürch AC, van Helden PD, Gagneux S, Warren RM and Gey van Pittius NC: Comparative analysis of Mycobacterium tuberculosis pe and ppe genes reveals high sequence variation and an apparent absence of selective constraints. PLoS One 7: e30593, 2012.

14. Copin R, Coscollá M, Seiffert SN, Bothamley G, Sutherland J, Mbayo G, Gagneux S and Ernst JD: Sequence diversity in the pe_pgrs genes of Mycobacterium tuberculosis is independent of human T-cell recognition. MBio 5: e00960-e13, 2014.

15. Boehme CC, Nabeta P, Hillemann D, Nicol MP, Shenai S, Krapp F, Allen J, Tahirli R, Blakemore R, Rustomjee R, et al: Rapid molecular detection of tuberculosis and rifampin resistance. N Engl J Med 363: 1005-1015, 2010.

16. Dong H, Liu Z, Lv B, Zhang Y, Liu J, Zhao X, Liu J and Wan K: Spoligotypes of Mycobacterium tuberculosis from different provinces of China. J Clin Microbiol 48: 4102-4106, 2010.

17. Kamerbeek J, Schouls L, Kolk A, van Agterveld M, van Soolingen D, Kuijper S, Bunschoten A, Molhuizen H, Shaw $\mathrm{R}$, Goyal M and van Embden J: Simultaneous detection and strain differentiation of Mycobacterium tuberculosis for diagnosis and epidemiology. J Clin Microbiol 35: 907-914, 1997.

18. Brudey K, Driscoll JR, Rigouts L, Prodinger WM, Gori A, Al-Hajoj SA, Allix C, Aristimuño L, Arora J, Baumanis V, et al: Mycobacterium tuberculosis complex genetic diversity: mining the fourth international spoligotyping database (SpolDB4) for classification, population genetics and epidemiology. BMC Microbiol 6: 23, 2006.

19. Larkin MA, Blackshields G, Brown NP, Chenna R, McGettigan PA, McWilliam H, Valentin F, Wallace IM, Wilm A, Lopez R, et al: Clustal W and Clustal X version 2.0. Bioinformatics 23: 2947-2948, 2007.

20. Hall, TA: BioEdit: A user-friendly biological sequence alignment editor and analysis program for Windows 95/98/NT. Nucl Acids Symp Ser 41: 95-98, 1999.

21. Ernst JD, Lewinsohn DM, Behar S, Blythe M, Schlesinger LS, Kornfeld $\mathrm{H}$ and Sette A: Meeting Report: NIH workshop on the tuberculosis immune epitope database. Tuberculosis (Edinb) 88: 366-370, 2008

22. Hsu T, Hingley-Wilson SM, Chen B, Chen M, Dai AZ, Morin PM, Marks CB, Padiyar J, Goulding C, Gingery M, et al: The primary mechanism of attenuation of bacillus Calmette-Guerin is a loss of secreted lytic function required for invasion of lung interstitial tissue. Proc Natl Acad Sci USA 100: 12420-12425, 2003.

23. Pym AS, Brodin P, Majlessi L, Brosch R, Demangel C, Williams A, Griffiths KE, Marchal G, Leclerc C and Cole ST: Recombinant BCG exporting ESAT-6 confers enhanced protection against tuberculosis. Nat Med 9: 533-539, 2003.
24. Stanley SA, Raghavan S, Hwang WW and Cox JS: Acute infection and macrophage subversion by Mycobacterium tuberculosis require a specialized secretion system. Proc Natl Acad Sci USA 100: 13001-13006, 2003.

25. Brodin P, Rosenkrands I, Andersen P, Cole ST and Brosch R: ESAT-6 proteins: Protective antigens and virulence factors? Trends Microbiol 12: 500-508, 2004.

26. Guinn KM, Hickey MJ, Mathur SK, Zakel KL, Grotzke JE, Lewinsohn DM, Smith S and Sherman DR: Individual RD1-region genes are required for export of ESAT-6/CFP-10 and for virulence of Mycobacterium tuberculosis. Mol Microbiol 51: 359-370, 2004.

27. Okkels LM, Brock I, Follmann F, Agger EM, Arend SM, Ottenhoff TH, Oftung F, Rosenkrands I and Andersen P: PPE protein (Rv3873) from DNA segment RD1 of Mycobacterium tuberculosis: Strong recognition of both specific T-cell epitopes and epitopes conserved within the PPE family. Infect Immun 71: 6116-6123, 2003.

28. Okkels LM and Andersen P: Protein-protein interactions of proteins from the ESAT-6 family of Mycobacterium tuberculosis. J Bacteriol 186: 2487-2491, 2004.

29. Teutschbein J, Schumann G, Möllmann U, Grabley S, Cole ST and Munder T: A protein linkage map of the ESAT-6 secretion system 1 (ESX-1) of Mycobacterium tuberculosis. Microbiol Res 164: 253-259, 2009.

30. Brodin P, Majlessi L, Marsollier L, de Jonge MI, Bottai D, Demangel C, Hinds J, Neyrolles O, Butcher PD, Leclerc C, et al: Dissection of ESAT-6 system 1 of Mycobacterium tuberculosis and impact on immunogenicity and virulence. Infect Immun 74: 88-98, 2006.

31. Vordermeier HM, Hewinson RG, Wilkinson RJ, Wilkinson KA, Gideon HP, Young DB and Sampson SL: Conserved immune recognition hierarchy of mycobacterial $\mathrm{PE} / \mathrm{PPE}$ proteins during infection in natural hosts. PLoS One 7: e40890, 2012.

32. Comas I, Chakravartti J, Small PM, Galagan J, Niemann S, Kremer K, Ernst JD and Gagneux S: Human T-cell epitopes of Mycobacterium tuberculosis are evolutionarily hyperconserved. Nat Genet 42: 498-503, 2010.

33. Parwati I, van Crevel $\mathrm{R}$ and van Soolingen D: Possible underlying mechanisms for successful emergence of the Mycobacterium tuberculosis Beijing genotype strains. Lancet Infect Dis 10: 103-111, 2010.

34. Kwan CK and Ernst JD: HIV and tuberculosis: A deadly human syndemic. Clin Microbiol Rev 24: 351-376, 2011

35. Mukherjee P, Dutta M, Datta P, Dasgupta A, Pradhan R, Pradhan M, Kundu M, Basu J and Chakrabarti P: The RD1-encoded antigen Rv3872 of Mycobacterium tuberculosis as a potential candidate for serodiagnosis of tuberculosis. Clin Microbiol Infect 13: 146-152, 2007. 